\title{
Performance assurance of mechanical engineering construction in the existence of cracks
}

\author{
Nikolay Velikanov ${ }^{1, *}$, Sergey Koryagin ${ }^{1}$, and Oleg Sharkov ${ }^{1}$ \\ ${ }^{1}$ Immanuel Kant Baltic Federal University Sciences, Institute of Engineering and Technology, 236016 Kaliningrad, Russian Federation
}

\begin{abstract}
The purpose of this paper is to consider the development of engineering ways of preventing the further development of through cracks in the sheet elements of the complex technical structures. The latest individual approach to the assessment and assurance of performance of structures with cracks, which is to take into account the features of their operation, has been applied in this work. Preference is given to methods that reduce the concentration in the danger zone. Studies have shown that the most appropriate and effective ways to prevent the further development of a through crack are the structural methods and combinations of the structural and technological methods. The effectiveness of these methods was determined on the basis of the strength uniformity approach of structural elements with a crack. The criterion of strength uniformity from the position of fatigue and brittle strength with an error on the safe side is the value of theoretical stress concentration factor $[K]$. The $[K]$ value was made on the basis of generalization and analysis of cases of crack formation, the results of tests of typical components of hull structures and welded joints. The safe concentration of stresses near the holes of various purposes in the members of the machine-building structures is in the range from 2 to 4 . Taking into account the relevant requirements for the ratio of the radius of curvature and the width of the cutout, we obtain 3.5. The value of the criterion $\mathrm{K} 1$ is determined by the ratio of the calculated crack length to the diameter of the hole drilled at its ends and the location of the crack.
\end{abstract}

\section{Introduction}

The use of fracture mechanics to assess the performance of machine-building structures made it possible to proceed to the normalization of various parameters characterizing the crack resistance of the material [1-9]. This made it possible to choose material in terms of preventing crack formation. However, structural and technological deficiencies often lead to the appearance of cracks in the nodes and parts of mechanical transmissions.

Existing computational methods do not provide simple and reliable algorithms to quickly assess the effect of various cracks on the performance of machinebuilding structures. Hence an individual approach to assessing and ensuring the performance of nodes and parts with cracks remains relevant. It takes into account the features of operation in machine drives [2-4].

In [1], the crack resistance of drive elements of wind turbine was investigated. Impact tests, chemical analysis, hardness tests, tensile tests, and fracture toughness tests were performed on specimens taken out of the three microstructures of the material. The influence of mechanical properties and crack resistance on critical estimates of the crack size is investigated. The results concerning the influence of material properties on structural design and structural assessment are discussed.
The study of the crack resistance of machine-building structures made of magnesium alloys is the subject of [2]. It is shown that the new technology of laser surface treatment, the laser shock treatment, promises to improve the technical characteristics of $\mathrm{Mg}$ alloys by increasing their surface strength, biocompatibility, fatigue resistance, and anti-corrosion ability. The process-microstructure ratio of $\mathrm{Mg}$ alloys was established during laser shock processing. The microstructures before and after laser processing and the effect of laser intensity on the microstructure were investigated.

The purpose of [3] is to describe the methodology for assessing the crack resistance of metal pipes due to corrosion damage. A gradation scale was developed representing the magnitudes of each defect type (dimples, pits, weight loss, and cracks). It allows to assess the need to repair or replace a part of the metal.

\section{Methods to ensure the performance of structures in the presence of cracks}

\subsection{Current state of the problem}

The existing methods of ensuring the performance of machine-building structures with cracks by using doubler plates welded along the contour, or cutting

Corresponding author: nvelikanov@kantiana.ru 
sections with cracks and welding of new plates, have the following disadvantages: the large volumes of welding and related work; additional concentration along the contour of the welding; gap corrosion; the possibility of the effect of the off-center loading; the presence of tensile residual stresses inside the welding contour.

Crack welding in most cases is also impractical for the following reasons.

The occurrence of cracks, as a rule, takes place in the area with increased stress concentration or in the defect area. After the crack grows, the area of its origin is unloaded and the zone of the crack's tip becomes the most dangerous. The welding of cracks leads to the restoration of the original pattern of stress distribution, and therefore to a dangerous concentration.

In addition, residual welding stresses that are tensile inside the heat-affected zone, as well as imperfections of the seam shape and possible embrittlement of the material can lead to an increase in the probability of cracking [3]. Therefore, it is advisable to give preference to methods that reduce the concentration in the danger zone.

Let us consider methods that can be applied in the case of the detection of equilibrium cracks in the nodes of the hull machine-building structures at the stage of choosing a quick and low-cost method of repair.

\subsection{Criteria and methods for assessing the performance of structures}

Studies have shown $[2,4,10]$ that the most appropriate and effective ways to prevent the further development of a through crack situated in a flat structural element during operation and repair are the structural methods (complete drilling of cracks, a system of unloading holes at the end of the crack, doubling plates installed without welding) and combinations of the structural and technological methods (welding seams and stiffeners, placed at a certain distance from the crack location).

The effectiveness of these methods was determined on the basis of the strength uniformity approach of structural elements with a crack, repaired by such methods and other structural elements made of the same material, operating under similar conditions, containing structural or technological stress concentrators permitted by the standards [1].

The criterion of strength uniformity from the position of fatigue and brittle strength of flat elements at a moderate level of nominal stresses (typical for the main members of the mechanical engineering design's hulls) with an error on the safe side is the value of theoretical stress concentration factor $[K]$.

Thus, in order to choose a way to prevent further crack development, it is necessary to determine the value of the theoretical stress concentration factor $K$ for the intended constructional or technological method, and also compare it with the acceptable value $[K]$ from the condition $K \leq[K]$.

The $[K]$ value was made on the basis of generalization and analysis of cases of crack formation, the results of tests of typical components of hull structures and welded joints; experimental and theoretical studies of stress concentration in hull connections.

The most provocative areas for occurrence of cracks are the angles of isolated and grouped cutouts. The safe concentration of stresses near the holes of various purposes in the hull elements of machine-building structures is in the range from 2 to 4 . The typical welded joints are characterized by similar interval of theoretical stress concentration.

The value $[K]$ was taken from $2 \ldots 4$ interval in accordance with the following. For hulls of machinebuilding structures it is allowed to make in shell plates the unsupported insulated cutouts having a width (diameter) of less than 20 thicknesses or $300 \mathrm{~mm}$, whichever is less. Taking into account the relevant requirements for the ratio of the radius of curvature and the width of the cutout, we obtain 3.5.

The value of the criterion for the specific abovementioned methods is determined primarily by the ratio of the calculated crack length to the diameter of the hole drilled at its ends and the location of the crack.

The cracking is explained in most cases not by the high level of stress macroconcentration (except for the presence of hard points) as much as by the combination of macroconcentration with microconcentration (technological concentration reflected by the effective concentration ratio) and such adverse factors as poor quality steel, low temperature, dynamic loads, etc. $[1$, $11-22]$.

\subsection{Calculation schemes}

The fatigue tests of specimens of machine steel [1] have shown that the processing of the reamer holes made at the crack tips allows, at a moderate level of nominal stresses, to eliminate almost completely the effect of microconcentration.

The diameter of the hole made by mechanical drilling with reamer in elements up to $20 \mathrm{~mm}$ thick under conditions of operation and repair of real structures is limited to about $50 \mathrm{~mm}$. Thus it is advisable to subdivide the cracks by the length criterion into short ones - up to $30 \mathrm{~mm}$, medium ones - up to $100 \mathrm{~mm}$, long ones - more than $100 \mathrm{~mm}$. According to the location criterion cracks can be of two types: the one passing the plate or hole's edges and the other cracks, conventionally called "insulated"

The most common types of cracks are shown in fig. 1-4. Full drilling can be used only for short cracks: insulated cracks (Fig. 1), cracks passing through the edge of the element (Fig. 2), cracks passing through the edge of a round hole (Fig. 3), cracks passing through the edge of a rectangular hole (Fig. 4). For the long isolated cracks, drilling with the installation of doubler plates can be used (Fig. 5).

The basic reference value for determining the value is the crack's effective length determined by the formula:

$$
l=c+m S,
$$


where $c-$ is the distance between the tips of an insulated crack, or the distance between the crack's tip and the element or hole's edge that are passed through by the crack; $S-$ is the thickness of the element at the tip of the crack; $m$ - is the empirical coefficient ( $m=1$ if the crack passes through the edge of the element or hole; $m=2-$ for an isolated crack).

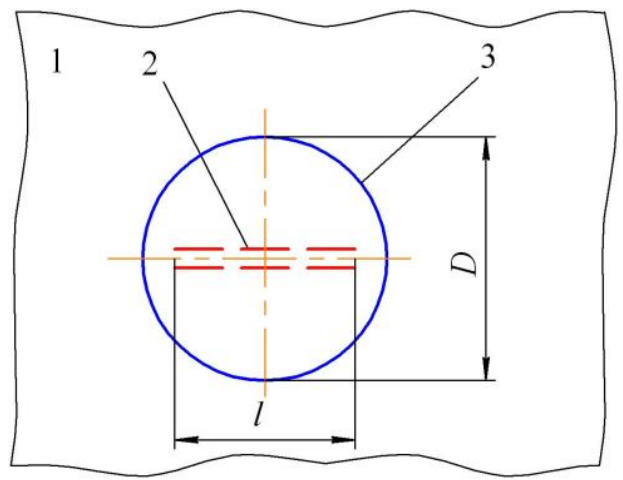

Fig. 1. Insulated crack: 1 - hull; 2 - crack; 3 -drilled hole.

When determining $c$ the distortion of crack path from the straight line is not taken into account, since the stress concentrator formed after drilling its ends, is almost equivalent to the concentrator of two holes connected by a straight section. The term $m S$ was introduced to have regard to the curvature of the front of the crack propagation through the thickness of the structural element and the plastic zone developing in the area of the crack tip.

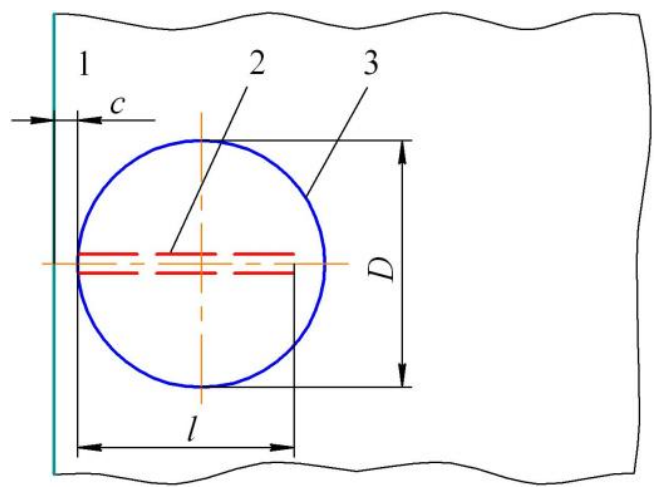

Fig. 2. The crack passing through the edge of the element: $1-$ hull; 2 - crack; 3 - drilled hole.

For machine-building structures with building thicknesses in the range of 6-18 $\mathrm{mm}$, the dimensions of the plastic zone in fracture mechanics are determined according to Irwin [1] and do not exceed the thickness of the metal.

The additional reference values are the diameter of the main hole $D$ made at the crack tip; width of the doubler plate $B$; diameter of the additional unloading hole $d$; the characteristic dimensions of the holes $D_{1}$ and $H$ through the edge of which the crack passes.

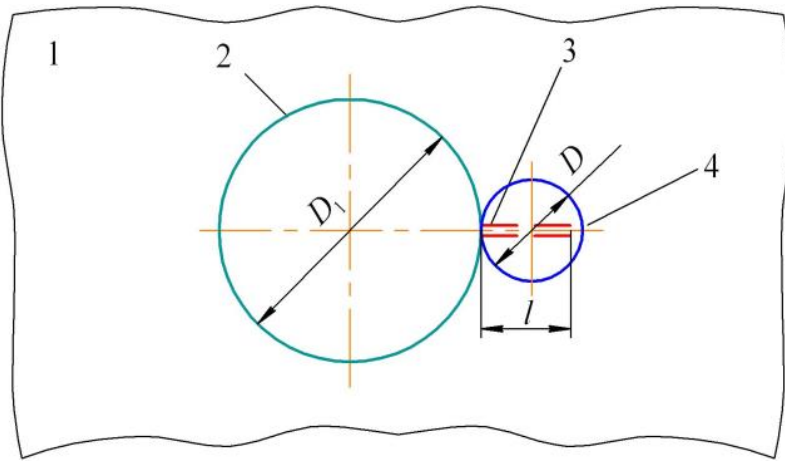

Fig. 3. The crack passing the edge of a round hole: $1-$ hull; 2 - structural round hole; 3 - crack; 4 - drilled hole.

After determining the value $l$ it is advisable to adhere to the following method choice sequence: complete drilling (Fig. 1-4), stop drilling of one hole, stop drilling of three holes; stop drilling with the installation of doubler plates (Fig. 5); combination of constructional and technological methods. In this case, unloading holes at the tips of the cracks are necessary for medium and long cracks.

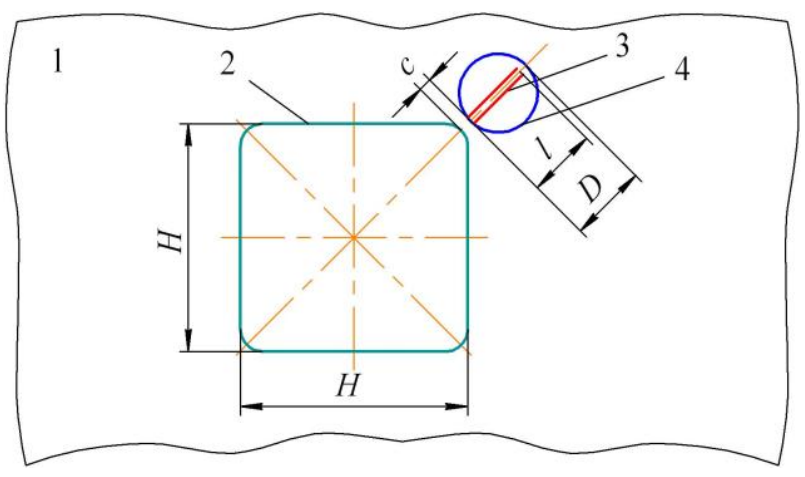

Fig. 4. The crack passing the edge of a rectangular hole: $1-$ hull; 2 - structural hole; 3 - crack; 4 - drilled hole.

The diameter of the drilled hole is determined by the formula

$$
D=l+S .
$$

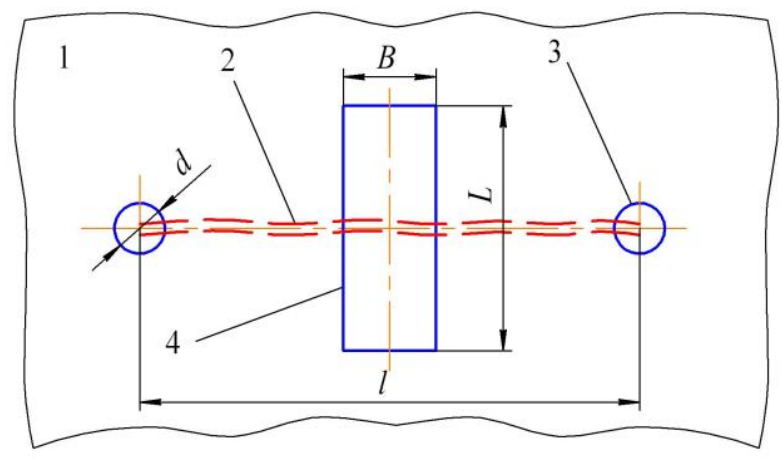

Fig. 5. Long crack: 1 - hull; 2 - crack; 3 - unloading hole; 4 - doubler plate. 


\subsection{Theoretical models}

Determination of $K$ coefficients for various concentrators represented by drilled cracks is performed either by well-known dependencies, or by the results of solving the corresponding problems with the methods of the two-dimensional elasticity theory, or experimentally $[1,3,23-29]$.

For short cracks, completely drilled with a hole with a diameter of $D=50 \mathrm{~mm}$, the values of stress concentration factors are as follows: a short crack passing the edge of the element $K=3.83$, an insulated crack $-K=3$.

If the crack passes the edge of a circular hole with a diameter of $D_{1}$, then

$$
K=\frac{1}{2}\left(1+\frac{D_{1}}{D}\right)+2 \sqrt{\frac{D_{1}}{D}+1}
$$

and if it passes the edge of a rectangular hole, with a size $H$, then

$$
K=1+2 \sqrt{\frac{H}{D}+1} .
$$

In the case of medium thicknesses, the additional unloading holes and doubler plates are used besides stop drilling of the crack ends. The diameter of the unloading hole is determined by the formula

$$
d=\frac{l}{2+(l / D)} \text {. }
$$

The width and length of the doubler plates is determined respectively by the dependencies:

$$
\left\{\begin{array}{l}
B \geq \frac{2 D+l}{6} \\
L \geq 2 B
\end{array}\right.
$$

Two additional unloading holes are placed next to the main one, slightly penetrating into it. The centers of the additional holes lie on the line passing through the center of the main hole and perpendicular to the direction of the crack. The doubler plate is mounted on rivets perpendicular to the direction of the crack at the edge of the element.

If the crack passes the edge of the element, then

$$
K=1+2 \sqrt{\frac{2 l}{D}+1}
$$

on the edge of a circular hole with a diameter of $D_{1}$

$$
K=\frac{1}{2}\left(1+\frac{D_{1}}{D}\right)+2 \sqrt{\frac{l+0.5 D_{1}}{D}+1} .
$$

When using two additional unloading holes

$$
K=0.62+1.24 \sqrt{\frac{2 l}{D}+1} .
$$

For crack passing through the edge of the element when using the doubler plate

$$
K=0.7\left(\frac{l-B}{D}\right)+2.65,
$$

for insulated crack

$$
K=1+2 \sqrt{\frac{l}{D}+1} .
$$

When using two additional unloading holes in the case of insulated crack

$$
K=0.62+1.24 \sqrt{\frac{l}{D}+1},
$$

when using the doubler plate

$$
K=0.35\left(\frac{l-B}{D}\right)+2.65 .
$$

In case of long cracks, the unloading holes made in the crack tip and the doubler plates are used.

For the crack passing the edge of the element, two doubler plates are used that are installed perpendicular to the crack direction. The first $B$ wide plate is placed near the unloading hole, the second $B / 2$ wide plate is placed at the edge of the element.

The figure 5 shows a nomogram for selecting the width of the doubler plate for the long crack, drilled with a hole $D=40 \mathrm{~mm}$.

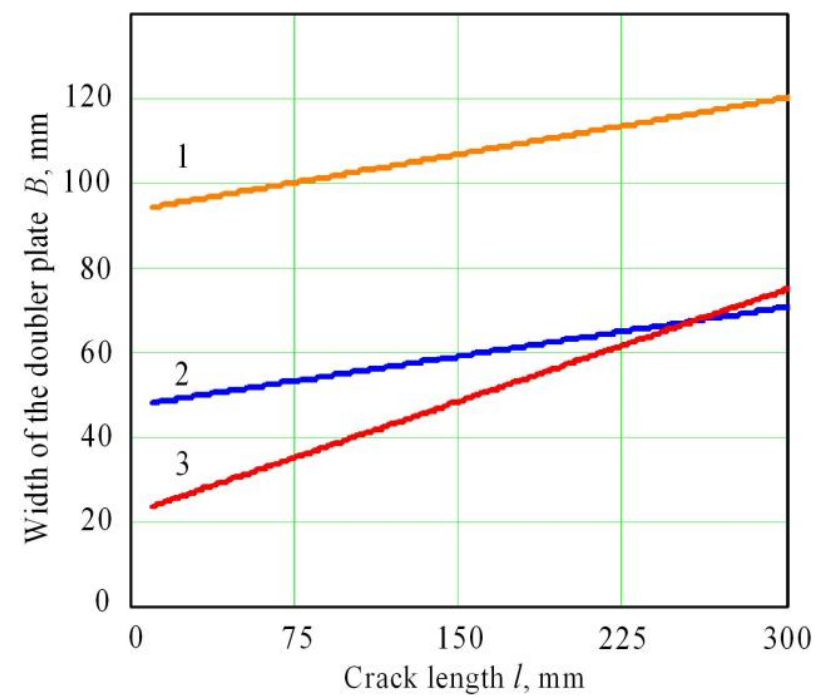

Fig. 6. Nomogram for selecting the width of the doubler plate: 1 - crack passing the edge of the element; 2 and 3 insulated crack with two and three doubler plates, respectively.

The following conditions must be fulfilled:

$$
\left\{\begin{array}{l}
B \geq \frac{5 D+2 l_{p}}{6} \\
L \geq 2 B
\end{array} .\right.
$$


For the insulated crack a stiffener is installed next to each of two unloading holes, with conditions

$$
\left\{\begin{array}{l}
B \geq \frac{5 D+l}{6} \\
L \geq 2 B
\end{array}\right.
$$

Or an additional third stiffener is installed between them with conditions

$$
\left\{\begin{array}{l}
B \geq \frac{14 D+l}{12} \\
L \geq 2 B
\end{array}\right.
$$

The installation of the doubler plates selected according to this nomogram and the formulas above, provides for the resulting concentrator values of $K=3.0$.

\section{Analysis of results}

The authors propose the following ways of preventing development of the crack: full drilling of the cracks, drilling of unloading holes at the crack tip, using the doubler plates. The formulas (3)-(16) and schemes presented in the article allow to provide the permissible value of the stress concentration coefficient for these methods.

\section{Conclusion}

Constructional methods of crack arrest associated with the stop drilling of their ends are effective for hull machine-building structures. They allow to avoid repair by replacement. For short and medium cracks, constructional methods reduce the stress concentration factor to acceptable values. For long cracks the doubler plates are used additionally.

\section{References}

1. A. Mehmanparast, J. Taylor, F. Brennan, I. Tavares, Fatig. \& Fract. of Eng. Mat. \& Struc. 41(12), 24852501 (2018)

2. B. Mao, Y.L. Liao, B. Li, Appl. Surf. Scien. 457, 342-351 (2018)

3. N. Cheriet, N.E. Bacha, A. Skender, Heliyon. 4(10), e00865 (2018)

4. N. Velikanov, S. Koryagin, O. Sharkov, Matec Web of Confer. 224, 01108 (2018)

5. D. Gornostajev, G. Aryassov, I. Penkov, Intern. Rev. of Mechan. Eng. 10(2), 115-124 (2016)

6. E. Dzindo, S. Sedmak, Z. Radakovic, I. Cvetkovic, Proc. Struct. Integ. 13, 420-423 (2018)

7. S. Lindqvist, T. Sarikka, M. Ahonen, H. Hänninen, Eng. Fract. Mechan. 201, 130-143 (2018)

8. Y. Javadi, M. Ashoori, Mater. and Des. 85, 82-90 (2015)
9. A.F. Liu Mechanics and Mechanisms of Fracture: An Introduction (ASM International, 2005)

10. N. Velikanov, S. Koryagin, O. Sharkov, J. of Phys.: Confer. Ser. 1015, 052028 (2018)

11. M. Junaid, F.N. Khan, K. Rahman, M.N. Baig, Opt. and Las. Techn. 97, 405-419 (2017)

12. H. Huang, S. Tsutsumi, J.D.Wang, L.Q. Li, H. Murakawa, Fin. Elem. in Anal. and Des. 135, 1-10 (2017)

13. A.N. Vasileiou, M.C. Smith, J. Balakrishnan, J.A. Francis, C.J. Hamelin, Nucl. Eng. and Des. 323, 309-316 (2017)

14. S. Seo, E.W. Huang, W. Woo, S.Y. Lee, Inter. J. of Fatig. 104, 408-415 (2017)

15. S. Hossain, G. Zheng, C.E. Truman, D.J. Smith Exper. Techn. 41(5), 483-503 (2017)

16. Yildirim H.C. Intern. J. of Fat. 101(2), 408-420 (2017)

17. S. Kainuma, M.Y. Yang, Y.S. Jeong, S. Inokuchi, A. Kawabata, D. Uchida, Eng. Fail. Anal. 79, 520537 (2017)

18. P. Yukhymets, A. Gopkalo, G. Zecheru, M. Mihovski, Inter. J. of Offsh. and Pol. Eng. 26(3), 272-277 (2016)

19. C.C. Osgood Fatigue Design: International Series on the Strength and Fracture of Materials and Structures (Elsevier, 2013)

20. P. Kumar, K. Prashant Elements of Fracture Mechanics (Tata McGraw-Hill Education, 2009)

21. S.S. Manson, G.R. Halford Fatigue and Durability of Structural Materials (ASM International, 2006)

22. J. Sule, S. Ganguly, H. Coules, T. Pirling, J. of Manuf. Proc. 18, 141-150 (2015)

23. K. Ikushima, S. Itoh, M. Shibahara, Weld. in the World. 59(4), 589-595 (2015)

24. Y.H. Fu, H.O. Zhang, G.L.Wang, H.F. Wang, J. of Mater. Proc. Techn. 250, 220-227 (2017)

25. F. Iwamatsu, K. Miyazaki, M. Mochizuki, J. of Pres. Ves. Techn., Trans. of the ASME. 137(4), 041402 (2015)

26. N. Liu, H.L. Ren, J.Z. Li, L.H. Jia, Advan. Mater. Res. 918, 95-100 (2014)

27. A. Maeda, Y. Jin, T. Kuboki, J. of Mater. Proc. Techn. 225, 178-184 (2015)

28. H. Chen, H. Ma, X. Chen, S. Jiang, H. Wang, J. of Fail. Anal. and Prev. 15(4), 563-570 (2015)

29. P. Vourna, A. Ktena, P.E. Tsakiridis, E. Hristoforou, NDT and E Intern. 71, 33-42 (2015) 